\title{
EL PODER MEDICO, DESDE LA SOCIOLOGIA*
}

Josep A. Rodríguez

La profesión médica, en castizo la clase médica, es la profesión por antonomasia. Su gran lucha por diferenciarse del brujo, del hechicero y del sacerdote, se vio premiada a partir de la Edad Media por el reconocimiento por parte del Estado de un status especial. La propia profesión era la única con derecho exclusivo a poner en tela de juicio su práctica curativa, y la única legalmente permitida para autoeducarse y decidir sobre las personas que la irían a ejercer '.

La avalancha del capitalismo en pro de la competencia y del laissez faire no afectaron de una forma directa a esa profesión; más bien lo contrario. Surge reforzada con el sistema capitalista, aliándose definitivamente con el Estado y consiguiendo de éste la persecución de cualquier tipo de intrusismo, y el derecho al control de su práctica y organización del trabajo. No es

- Eurot Fremson, la profesión médica, Península, Barcelona, 1978, 375 págs.; Jesús M. DE Miguer, La reforma sanitaria en España, Cambio 16, Madrid, 1976, 262 págs.; Benjamfin Gonzál.zz, El capital humano del sector sanitario, Centro de Investigaciones Sociológicas, Madrid, 1979, 115 págs.

1 Para un planteamiento general conviene leer la introducción de HENRY SIGERIST a su monumental A History of Medicine, concretamente el volumen 1: Primitive and Archaic Medicine, Oxford University Press, Nueva York, 1951. 
un proceso lineal, y los poderes de la profesión médica varían de unos países a otros, dependiendo de los distintos procesos de afianzamiento y desarrollo del capitalismo. Luego veremos lo que sucede en España.

Hoy en día la profesión médica, en los países de más alto nivel económico, tiene un status que la convierte en autónoma con respecto al resto de la sociedad y en cabeza de la organización sanitaria. Tal es su importancia que las profesiones paramédicas tienen legitimado su status únicamente en función de su relación (de subordinación y dependencia) con el médico. La profesión médica no sólo controla exclusivamente su propio trabajo, sino que además controla y organiza el trabajo de otras profesiones que se establecen alrededor de ella en su labor de asistencia.

De sus relaciones con el poder establecido, o sea con el Estado, la profesión médica ha sido uno de los grupos profesionales más claramente favorecidos. Su autonomía y el poder que ésta le otorga es utilizada para la generación de más poder, convirtiéndose así en una de las «sociedades paralelas» de más influencia en nuestra sociedad ${ }^{2}$. Por otro lado, la profesión es uno de los vehículos ideológicos más importantes del Estado. Aplica y desarrolla la concepción del Estado acerca de la salud; pero a su vez influve en el Estado al hacerle asumir sus propios criterios y concepciones de lo normal y lo anormal. Se convierte así en, no únicamente el bacedor de la enfermedad, sino también el grupo social que dictamina sobre el bien y el mal en nuestra sociedad. Si a todo ello le unimos su poder e interés en lo económico no resulta extraña la calificación que de «empresario moral» hace de ella Freidson. Empresario de la moralidad que significa el monopolio sobre lo que es normal y sobre lo que es desviado, y monopolio de las técnicas a utilizar para solucionar dicha desviación. La profesión médica, al elevarse sobre el bien y el mal, se convierte de nuevo en el grupo social que interpreta las legendarias y divinas leyes que rigen el comportamiento del ser humano ${ }^{3}$.

La profesión médica, por su importante papel relacionado con la sociedad (su labor de asistencia curativa) es uno de los elementos más claros de estratificación social. En los países en los que priva la práctica individual y privada sobre la pública su dedicación a las clases pudientes de la sociedad es palpable. A excepción del médico humanista, y altruista, que todavía cree en aquella raída idea de que la medicina está al servicio de la humanidad, la mayoría de la profesión médica está preocupada en acrecentar su poder económico y su prestigio tanto científico como social. Es incluso un elemento de estratificación social en aquellas sociedades en las que la práctica pública es más importante que la privada; el caso español es un buen ejemplo de ello.

${ }^{2}$ En Europa esto empezó a analizarse sociológicamente a partir de Yvo NuYENs, Sociologie en Gezondheidszorg, Uitgeverij de Nederlansche Boekhandel, Amberes, 1969, 260 págs.

${ }^{3}$ Para el caso español hay que leer la aportación de George M. Foster, que aparece en Michael KenNy y Jesús M. DE Miguel (eds.), La antropología médica en España, Anagrama, Barcelona, 1980, págs. 123-147 y 249-265. 
La distribución regional de profesionales señala que los intereses mayoritarios del médico están ligados a la población que posee recursos económicos. La abundancia de médicos en las zonas desarrolladas, en las grandes ciudades, y en las zonas de abundancia de fortunas familiares corroboran la idea de que el médico prefiere hacer medicina para ricos que para pobres. $O$ como dicen los italianos: La medicina es aquella ciencia que dice al pobre cómo podría curarse si fuera rico.

\section{Poder tirano}

El libro de Freidson sobre la profesión médica ${ }^{4}$ es uno de los más importantes estudios de carácter teórico sobre la profesión médica. Su relevanncia no se limita al campo de esta profesión sino que se extrapola al campo de la sociología de las profesiones, ocupando un lugar importante en su biblioteca de autores. El autor pretende, a través de este análisis de la profesión médica, contribuir a la comprensión tanto de la estructuración y organización de la profesión médica como de cualquier profesión. La meta a la cual quiere aportar su trabajo es al conocimiento de la organización, opiniones, y prácticas específicas de cada profesión, dejando de lado sus conocimientos específicos.

El minucioso análisis de la profesión médica demuestra la utilidad de conceptualizar a una profesión como un tipo de organización ocupacional, en la cual domina un esquema mental, y que es capaz, dado su rol dominante en la sociedad, de transformar e incluso crear su propio trabajo. Por ello, inicia su exposición con los elementos que hacen diferente a una profesión de cualquier acupación. La medicina logra un status especial al convertirse en predominante en una compleja división del trabajo, encontrándose libre para controlar no sólo el contenido de su labor sino incluso los límites mismos de su trabajo.

Se centra en el estudio de las instituciones médicas norteamericanas, y en concreto de los distintos marcos en los cuales desarrolla su práctica, el control interno de la misma, y la configuración de su organización. Llega así al objeto de la medicina: la enfermedad, como concepto social, es decir haciendo referencia a la desviación de la normalidad.

En tanto la medicina no fue una ocupación cuyo conocimiento y ciencia no adquirieron un elevado nivel, falló en conseguir las condiciones básicas para desarrollarse como una profesión-de-consulta, con un status verdaderamente profesional. En muchos aspectos mantenía todavía similitudes con el brujo y el hechicero. No fue hasta la Edad Media en que empieza a adquirir

4 Errot Fremson, La profesión médica: Un estudio de sociología del conocimiento aplicado, Península, Barcelona, 1978, 375 págs. El prefacio (págs. 9-33) es especial para esta edición. El libro se publicó en inglés por primera vez en 1870. 
un status diferenciado del hechicero, al garantizársele por parte del Estado una competencia exclusiva para determinar el contenido adecuado y el método efectivo para tratar al enfermo. Pero, sin lugar a dudas, el elemento que la convertiría en una profesión-de-consulta no fue el status legal sino el desarrollo de destrezas y conocimientos que le permitirían, por primera vez, la predicción y el control seguro de un amplio espectro de enfermedades humanas. Con esa sólida base técnica el médico pudo gozar de confranza, demostrando la justicia de sus demandas de privilegio y monopolio de su conocimiento, convirtiéndose en profesión con autonomía.

La autonomía técnica, o sea el control profesional sobre el trabajo, es un elemento definidor de profesión. Este control tiene un carácter netamente político, pues surge de la relación con el Estado y es producto del interés de éste en el establecimiento y mantenimiento de la profesión. Su característica más importante se debe pues al Estado, del cual la profesión no es completamente autónoma. El Estado deja en manos de la profesión el control sobre los aspectos técnicos de su trabajo, y sobre la organización social y económica del mismo.

No es únicamente el control sobre su práctica sino además el control sobre la organización y división del trabajo lo que hace que la medicina sea distinta de otras profesiones. Está situada en el vértice de la organización de la asistencia sanitaria, y bajo su autoridad se encuentran los profesionales paramédicos, con falta de autoridad, de autonomía, y con un prestigio relativamente bajo. La subordinación a la autoridad de la profesión médica, y el tener como única fuente de legitimización de su trabajo la relación con dicha profesión, son el origen de grandes conflictos. Cada día cuesta más encontrar personal que ingrese en dichas ocupaciones, dado el largo período de entrenamiento necesario y su escasa autonomía y prestigio profesional. La lucha por la ampliación de autonomía, prestigio y responsabilidad están afectando de una forma directa la estructura de la organización del trabajo médico ${ }^{5}$.

Las características formales de la profesión de la medicina son su derecho al control de su propio trabajo, y el derecho exclusivo a determinar quiénes pueden legítimamente ejercer su trabajo y cómo debiera realizarse éste. La profesión médica tiene derecho para declarar las valoraciones exteriores a ella como ilegítimas e intolerables. Esa posición privilegiada es concedida por la sociedad. Es esencial para su supervivencia que la élite dominante siga creyendo en el valor positivo de la profesión, para que la siga protegiendo frente a cualquier clase de intrusiones.

s El mismo autor ha tocado el tema en otras obras importantes: EuIot Freinson y Judrth Lorber, Medical Men and Their Work, Aldine, Chicago, 1972, 482 págs., y Eliot Freidson, Doctoring Together: A Study of Professional Social Control, Elsevier, Amsterdam, 1975, 298 págs. 
El ambiente cotidiano de trabajo profesional es muy variado, pasando de una práctica completamente individual y aislada, por la práctica de colegas, de asociación, la pequeña sociedad legal, y la práctica hospitalaria. Básicamente son dos tipos de práctica: la individual, aislada del contacto con los colegas y con posibles deficięncias de tipo cualitativo, que es una práctica dependiente del paciente; y la de cooperación con colegas, en la cual la práctica deppende de éstos más que del cliente.

Podemos considerar dos tipos de práctica hospitalaria: la ligada directamente a la práctica privada y la hospitalaria en sí. Cuando es una extensión literal de la consulta privada, el hospital se convierte en el lugar donde se tratan casos poco usuales pero rutinarios. Todos los tipos de práctica médica en las sociedades industrializadas incluyen acuerdos y pactos con el hospital por los que el médico lo utiliza como lugar donde internar y tratar pacientes. Cuando hablamos de prácticas hospitalarias en sí podemos diferenciar dos tipos de médicos: los MIR (internos y residentes) que están en plena fase de formación necesaria para la adquisición de su pericia profesional, y los médicos de plantilla.

El médico se encuentra en el hospital con un trabajo organizado independiente de su práctica individual. Planteando demandas, aludiendo al bienestar del paciente, invocando amenazas sobre su vida y proclamando su capacidad para evaluar y solucionar los problemas, es lo que permite al médico enfrentarse y no quedar subordinado por la línea de poder administrativo y jerarquizada del hospital. Ello genera otra línea de poder, no-oficial ni jerarquizada, la representada por los médicos.

En organizaciones relativamente complejas como los hospitales no existen mecanismos estables de autorregulación profesional. Los médicos se rebelan fuertemente a cualquier tipo de restricción que afecte la independencia de sus decisiones. Cuando hay transgresores a las normas se utiliza la técnica de exclusión o boicot personal. Es un intento de impedir que un profesional trabaje con ellos o con sus pacientes, pero nunca de impedir su ejercicio profesional (simplemente de limitarlo a otras zonas, otros profesionales u otras especialidades).

Frente al concepto altruista que Parsons creó para definir el rol de la profesión, va surgiendo una práctica diaria que contradice dichos supuestos. El propósito del médico no es el conocimiento, sino la acción. Su amor por la acción le lleva en muchos casos a la utilización de medicamentos que sobrepasan lo indicado por las normas médicas y científicas. Es pragmático, se apoya más en resultados aparentes que en teoría; y por tanto tiende a confiar preferentemente en su propia acumulación de experiencia de primera mano: el «olfato» médico. La autoridad de sus sentidos es para él de mayor importancia que la tradición o la ciencia. Ello conduce a la existencia de prácticas inefectivas o insidiosas, que se prolongan por mucho tiempo. Dado que el médico proviene, generalmente, de la burguesía, enfatiza la indepen- 
dencia, el individualismo social y económico, y la dignidad de clase en su status.

La medicina ha obtenido una jurisdicción casi exclusiva para determinar qué es enfermedad y por tanto cómo debe actuar la gente para ser tratada como enferma. Está pues comprometida ẹn la creación de la enfermedad, como estado social que el ser humano puede asumir, creando las posibilidades sociales para su representación. La enfermedad es un tipo de desviación de un conjunto de normas que representan la salud y la normalidad; y la medicina se convierte en actividad moral que descubre aquellas cosas que son indeseables. La enfermedad, como un estado social, implica cambios en la conducta; precisamente cuando el médico diagnostica la enfermedad cambia la conducta del hombre.

Los presupuestos de Parsons acerca del enfermo y la enfermedad siguen siendo vigentes. Al enfermo no se le hace responsable por ello, se le libra de sus obligaciones normales, se le permite desviarse legítimamente, y se espera que busque ayuda para su recuperación. El rol del enfermo funciona para evitar que éste se convierta en un alienado permanente. A la enfermedad se le asigna una legitimidad condicional como desviación. Es condicional sólo en la medida en que se piensa que la recuperación es posible, y esta legitimidad está condicionada a que el enfermo trate de recuperarse ${ }^{6}$.

La salud es valorada porque es condición esencial para el desempeño adecuado de las tareas y roles asignados. El profesionalismo de la salud se erigió para legitimar la reivindicación de que la conducta adecuada ante la desviación es el tratamiento por profesionales responsables. Esto ha dado lugar al fortalecimiento de una institución de control, con carácter profesional (la profesión médica) que en nombre del individuo y de la pericia técnica, puede quitarle al profano el derecho para evaluar su propia conducta y la de sus compañeros. El médico se está convirtiendo en el empresario moral de la sociedad, que dicta lo que es normal o no, y lo que hay que hacer para co. rregir dicha desviación.

Por otro lado, la conducta del médico ante la enfermedad constituye la objetivización de los valores dominantes en la sociedad. El médico conduce el estado social de estar enfermo, dictamina si uno está o no enfermo, si debe adoptar el rol de enfermo, y si puede volver a asumir su identidad y status normal. La profesión está tomando decisiones sociales al diagnosticar la enfermedad, y es precisamente ese papel social el que la hace capaz de crear nuevas necesidades (en este caso nuevas enfermedades) para mantenerse en su posición de poder y privilegio.

Freidson duda de que el contenido de la profesión tenga uniformemente un carácter tan confiable científicamente como para justificar su exigencia de

- Véase su celebrado "capítulo diez", TAlcott Parsons, The Social System, Free Press, Nueva York, 1951, págs. 428-479. Hay una excelente traducción al español en Revista de Occidente. 
autonomía para todo el contenido de su trabajo. El diagnosticar la enfermedad es un compromiso moral; implica la indeseabilidad de ciertos procesos que influyen en la persona que los posee, y le aíslan de la sociedad. Dicha calificación de enfermedad no se basa únicamente en un fundamento de carácter científico. El médico puede demostrar que ciertos signos siguen determinado curso o que conducen a determinadas consecuencias; pero que sea malo o indeseable es algo que debe ser decidido por el conjunto de la sociedad, y no únicamente por el médico. El (o ella) no posee una capacitación especial para ser un experto exclusivo acerca de lo deseable o indeseable ${ }^{7}$.

La obra de Freidson tiene como objeto de estudio la profesión médica estadounidense. Muchas de sus hipótesis o conclusiones no son fácilmente extrapolables al conjunto de la profesión médica mundial. A pesar de la importancia, su obra tiene que ser matizada cuando se aplica a España. Precisamente ése es uno de los objetivos del presente artículo.

Para Freidson la esencia de la profesión médica es su autonomía, que se refleja en su derecho al control de su propio trabajo, y en el derecho exclusivo a determinar quiénes pueden legítimamente ejercerla. El control sobre su propio trabajo es un elemento de carácter universal, y es indudablemente el elemento diferenciador. Sin embargo, el derecho exclusivo a determinar quiénes pueden ejercerla y el derecho a la organización de su trabajo no son características tan universales. Depende en parte de la relación entre el Estado y la profesión, y en definitiva de la fuerza y cohesión de la profesión. En casos como el español, en que los Colegios de Médicos han tenido poco poder, y en que la mayoría de los médicos trabajan para el Estado (el 85 por 100 para el INSALUD), es este último quien decide quién puede o no ejercer la profesión. Es el Rey quien otorga la licenciatura, y el Estado quien al convertirse en contratador el que organiza el trabajo del médico. Indudablemente ello merma el control del médico sobre su propio trabajo y la profesión pierde poder y prestigio (comparándola con la profesión norteamericana).

La profesión médica se articula como grupo de presión en todas las sociedades. Dado su importante papel en las tareas del Estado, resalta en el trabajo de Freidson la ausencia de un estudio de dichas tareas y en definitiva el análisis de la profesión como organismo de poder. Estos estudios son numerosos en los países anglosajones y post-industriales, pero muy escasos en España ${ }^{8}$.

La profesión, como vehículo de estratificación social, es un elemento de gran importancia en cualquier estudio de los médicos. Es notable que no

7 Compárense estas ideas con Jean-Claude Polack, la médecine du capital, François Maspero, París, 1972, pág. 223.

* Todavía el mejor estudio sigue siendo el de HARRY Eckstern, Pressure Group Politics: The Case of the British Medical Association, Stanford University Press, Stanford, 1960, 168 págs. De interés en Whliam E. Steslicke, Doctors in Politics: The Poritical Life of the Japan Medical Association, Praeger, Nueva York, 1973, 303 págs. 
aparezca en el presente y más teniendo en cuenta que es en los Estados Unidos donde, con una práctica mayoritariamente privada, los efectos de dicho proceso serían más destacables.

La concepción de la enfermedad como desviación social, proviene de Parsons, y es propia de una sociedad altamente desarrollada como la norteamericana. Está, sin embargo, imbuida de un neto espíritu funcionalista. Es por ello que no se puede hacer extensible a todo el mundo, y más sabiendo que la concepción de la enfermedad está estrechamente ligada a la cultura de cada país. El enfermo, como desviado social, es una imagen típica de sociedades altamente desarrolladas a nivel económico. Es importante, sin embargo, la aportación a la enfermedad en tanto que construcción social. Es innegable que cada sociedad y cada cultura determina lo que es normal y lo que no lo es, y por tanto lo que es enfermedad.

No es el análisis de la posición actual de la profesión médica lo importante en el presente estudio, sino el temible futuro que Freidson plantea cuando predice que la profesión médica se convertirá en el empresario moral de la sociedad. Decidirá, por encima de toda la sociedad y población, qué es lo normal o lo no normal, y qué se puede hacer contra la anormalidad. Para evitar que la profesión se convierta en lo que en otros momentos históricos fue la Religión o el Derecho, Freidson plantea limitar su autonomía, tanto en base a criterios de tipo técnico como de tipo social y político. La profesión no tiene ninguna exclusividad en el conocimiento de lo que es deseable o no, y por tanto no se puede erigir en el único grupo social que dictamine acerca de la normalidad de nuestras vidas. El esquema teórico, en este sentido, es aplicable al caso español, aun cuando la «tiranía» profesional haya sido menor en las últimas décadas. Desde 1975 somos testigos de un renacimiento de esas prácticas de monopolio, autonomia, y definidores de la normalidad.

\section{La razón del poder}

En el caso concreto de nuestro país, hay dos obras que analizan el llamado capital bumano del sector sanitario. La primera de ellas, publicada en 1976, con el pretencioso título de La reforma sanitaria en España ${ }^{9}$, es un «libro blanco» de la sanidad española, y de ahí su articulación en párrafos numerados. La elaboración del mismo parte de la creencia de que: «los sociólogos si quieren abandonar su papel de contempladores de la escena social desde su torre de marfil deben involucrarse cada vez más en la ingeniería social, es decir, en el ejercicio de una sociología que lleve a políticas específicas para lograr el cambio social» ${ }^{10}$. Como todo libro blanco está compuesto

- Jesús M. DE Miguel, La reforma sanitaria en España: El capital humano en el seçtor sanitario, Cambio 16, Madrid, 1976, 262 págs. Es un proyecto realizado para la Dirección General de Sanidad, entre febrero y junio de 1976.

10 Ibídem, pág. 9. 
básicamente de dos partes: la primera es un análisis crítico de la situación, en el presente caso el estudio del capital humano en el sector sanitario, sus características más específicas, su distribución territorial y su estructuración; y en segundo lugar se plantean las alternativas para una reforma sanitaria en España.

El estudio se realizó en un momento crucial tanto para la sociedad española como para el sector sanitario español. Con los últimos coletazos de la época desarrollista, que no trajo aparejada una mejora relativa de la salud de la población, más bien lo contrario; y la entrada en unos años marcados por el inexorable peso de la crisis económica, aparece el señuelo de la democracia y por tanto de la racionalización y la democratización. El sector sanitario se enfrenta entonces con el desarrollo del mismo y el deterioro de la salud de la población ".

El capital humano del sector sanitario en España nunca ha sido objeto de una adecuada planificación, de ahí las considerables incongruencias que el sistema plantea. Considerando a España dentro del contexto europeo cuenta en 1976 con una baja tasa de personal sanitario: 293 por 100.000 habitantes frente a las tasas de 302 de Portugal, 512 de Italia, y 499 de Yugoslavia (paises todos ellos de un parecido nivel socioeconómico). A grandes rasgos se comprueba el relativo exceso de médicos y farmacéuticos, y la escasez de odontólogos, enfermeras, personal docente y camas en establecimientos sanitarios. Según el censo de 1970 uno de cada cuatro técnicos de grado medio y superior se encontraba formando parte de la población activa en el sector sanitario, lo que representaba el segundo sector social empleador de capital humano, con muchos técnicos de grado superior y medio cualificados y con experiencia.

Frente al crecimiento constante de la tasa de médicos ( 3 por 100 anual para el quinquenio 1968-1973, con posible incremento de dicha tasa en años sucesivos), profesionales como veterinarios y practicantes están en un desceso relativo. Los veterinarios porque todavía no han sido integrados en el sector alimenticio e industrial, y matronas y practicantes al perder espacio profesional frente al ATS. Dentro de la profesión médica observamos un decremento relativo de la tasa de médicos generales (del 49 por 100 en 1956 al 43 por 100 en 1970) y un incremento relativo de los especialistas (sobre todo pediatría y psiquiatría).

El sector farmacéutico es de gran importancia en España dada la importancia del gasto farmacéutico en el sector público. En 1965 el gasto farmacéutico representaba el 35 por 100 del total del gasto sanitario, y en 1970 ya

"El mismo tema se repite desde Salustuno del Campo Urbano, Problemas de la profesión médica española: Informe preliminar, Comisión Nacional Española del Instituto Internacional de Estudios de Clases Medias, Madrid, 1964, 85 págs.; hasta Jordr Gor I Gurina y cols., El metge de capçelera en un nou sistema sanitari, Laia, Barcelona, 1979, 178 págs. 
representaba el 50 por 100 . A pesar de ello, y de ser una profesión con un número elevado de miembros, su práctica está centrada al servicio directo del público (servicio de venta de fármacos) sin cubrir las necesidades en hospitales o en investigación.

El problema de la falta de enfermeras es de carácter mundial. A pesar de su rápido crecimiento, todavía en España por cada 100.000 habitantes hay más médicos y farmacéuticos (205) que ATS (174). A ello hay que añadirle el problema de la estabilidad profesional, más alta para médicos y farmacéuticos, pero con una gran brecha para las ATS entre los 26 y 37 años, pues son los años ligados a las épocas de matrimonio y maternidad. En España la enfẹtmería es una profesión netamente femenina, frente a medicina en la que la participación de las mujeres es baja. Durante años España fue el país industrializado del mundo con menos proporción de médicas. Farmacia tiene un alto porcentaje de mujeres en sus filas ( 75 por 100), mientras que veterinaria se destaca por su escasez (menos del 5 por 100 de los estudiantes).

Las características básicas de la enseñanza sanitaria en nuestro país son: falta de coordinación de recursos educativos con las necesidades reales sanitarias, y falta de una planificación y control de la producción de graduados. Prueba de ello es el excesivo ritmo de crecimiento del número de estudiantes de medicina, que ha hecho que durante años fuese mayor el número de estudiantes que el de los propios médicos. Mientras tanto, todavía se creía, $y$ se cree, en la falsa idea de falta de médicos.

Actualmente uno de cada cuatro estudiantes y uno de cada cinco licenciados es de ciencias médicas. El número de estudiantes en farmacia y veterinaria crece despacio, mientras que medicina y ATS están en continuo auge. A pesar del rápido incremento de estudiantes de ATS, todavía existen dos estudiantes de medicina por cada uno de ATS.

El gran incremento del número de estudiantes no corre paralelo ni con la ampliación de la plantilla de profesores (como clara protección de los intereses de los catedráticos) ni con la mejora de la calidad de la enseñanza. Todo ello ha conducido al deterioro de la calidad de la enseñanza impartida, y a una progresiva separación de la realidad, que ha conducido a una educación que en algunos casos es irrelevante.

El numerus clausus no ha servido todavía para frenar la gran avalancha de estudiantes (hasta 1984 no va a ser posible observar los efectos de éste sobre la profesión médica) y sí, en cambio, para incrementar las diferencias de carácter social y económico. Sólo el 6 por 100 de los estudiantes de medicina proviene de la clase trabajadora (que representa el 36 por 100 de la población activa). Medicina y farmacia son claramente facultades para las clases altas, y básicamente de origen urbano. Las tasas de mujeres estudiantes en ciencias médicas son las más bajas de Europa. En medicina representan el 30 por 100 de los estudiantes, en ATS sobrepasan escasamente el 
50 por 100 y en farmacia son del orden del 57 por 100 de los estudiantes y del 64 por 100 de los licenciados.

El personal sanitario que trabaja en hospitales ha triplicado su número entre 1949 y 1972. Ello da idea de la importancia que está adquiriendo el sector hospitalario en la medicina del país. Se ha incrementado mucho el número de médicos en el sector, llegando en 1973 a ser el 63 por 100 del total de médicos. Este desarrollo es debido, en primer lugar, a la gran expansión de los hospitales públicos frente a los privados, al mal estado de los hospitales y a su deficiente organización. En este sector la tónica es la misma que en el conjunto de la sanidad española: relativo exceso de médicos, y deficiente número de enfermeras. Como elemento a destacar de este sector es la superioridad de la medicina hospitalaria pública sobre la privada.

En España la población activa del sector sanitario muestra una clara tendencia a concentrarse en las regiones con mayor nivel económico, y en las ciudades y centros urbanos más importantes. La concentración de personal sanitario en el litoral, centro y vértices del hexágono del desarrollo, coincide con la distribución del desarrollo económico y la urbanización; y por tanto con las pautas norte-sur y este-oeste. Norte y este con abundancia de personal sanitario, y sur y oeste con alarmante escasez. Para una población urbana que representa el 52 por 100 del total de la población española hay una población sanitaria del orden del 86 por 100 de los ATS y 78 por 100 de los médicos y farmacéuticos; para la población rural (29 por 100 del total) sólo corresponde el 6 por 100 de los ATS y el 11 por 100 de los médicos y farmacéuticos. Ello muestra que las profesiones sanitarias son básicamente urbanas. La población sanitaria se concentra en las capitales de provincia; la media nacional es de 3,4 veces más profesionales en la capital que en el resto de la provincia. En 1972 ya el 66 por 100 de los médicos ejercían en capitales de provincia.

La existencia de Facultades de Medicina, Farmacia y Veterinaria es un claro elemento de atracción de profesionales sanitarios. La concentración alrededor de las mismas es clara. Con algunas excepciones, la distribución regional de facultades sigue también la pauta de desarrollo-urbanización. Madrid y Barcelona son siempre grandes polos de atracción de profesionales, dado su carácter de centros económicos y administrativos.

El médico general se concentra en el norte y el médico especialista lo hace en Madrid, el litoral, y con una muy alta proporción en el sur. La pauta de distribución de especialistas sigue la de urbanización, con tendencia hacia el litoral, y con alta concentración en Barcelona y Madrid. En el sur de la Península se practica una medicina para ricos, mientras se desatiende a la mayoría de la población; prueba de ello es la escasez de médicos generales. La concentración de especialistas en las capitales de provincia es superior a la de médicos, es decir 3,5 veces más que en el resto de la provincia. Las ATS 
siguen las mismas pautas que los médicos: excesiva concentración en zonas urbanas y escasa dedicación a la medicina rural.

En general, los farmacéuticos están concentrados en las regiones menos desarrolladas, con la excepción de Madrid y Barcelona. La alta concentración de farmacéuticos en el sur y el elevado gasto farmacéutico de la zona hacen dudar de la calidad asistencial.

La pauta que domina la distribución del personal sanitario en hospitales es la de los ejes norte-sur y este-oeste. El norte y el este tienen abundancia relativa de personal, mientras que el sur y el oeste tienen escasez. Las provincias con facultades de ciencias médicas son las que tienen una relación camas-médicos más favorable. Frente a un norte-este con una práctica médica relativamente aceptable, el sur español, Galicia y un amplio sector de Castilla la Nueva se encuentran en una situación sanitaria deteriorada y ello debido básicamente a su posición marginal con respecto a los procesos de desarrollo socio-económicos. Ha sido precisamente en estas zonas donde se ha producido en los últimos años una «falsa redistribución" por la cual algunas zonas más subdesarrolladas han visto crecer sus tasas de personal sanitario a mayor velocidad que la media nacional. Ello ha sido debido a: un punto de partida más bajo, una progresiva urbanización del litoral, y a la errónea redistribución regional, que se ha realizado a nivel provincial y no a nivel de zonas rurales-urbanas o teniendo en cuenta la distribución de la renta.

Según esta argumentación, España necesita de una drástica reforma del sector sanitario que reduzca las desigualdades de tipo socio-económico, y que conduzca a un igual nivel de salud para toda la población. Una de las razones fundamentales para ella es que su coste es menor que el de la no-reforma, en tanto que una mejor organización del capital humano del sector sanitario podría disminuir el gasto total por paciente.

El cambio propuesto parte de un sistema que haga que todos los ciudadanos reciban los cuidados positivos de prevención, curación y rehabilitación, a través de un sistema único; en base a la creación de un servicio sanitario nacional que, con cargo a los presupuestos del Estado, garantice a toda la población un nivel adecuado de salud y sirva a su vez como sistema de redistribución de recursos dentro de la sociedad. El capital humano del sector sanitario es una de las piezas claves del mismo y por tanto necesita también de una reestructuración, por la cual se cubra el triple aspecto de la medicina preventiva, curativa y rehabilitadora, y conduzca a la unificación de dicho capital a través de una eficaz reorganización y planificación de los recursos humanos. Los dos objetivos suponen la superación de la dicotomía entre sector público y privado, y la organización de la educación sanitaria de la población, para hacer posible su colaboración en el sistema sanitario.

A pesar de ser un libro publicado en 1976 , y con la mayoría de datos referentes al año 1974, es una obra con persistente actualidad. Han pasado cinco años desde su publicación y la situación del sector sanitario español es 
muy similar. Ello prueba que los esfuerzos intelectuales por of recer soluciones a los problemas sociales no son todavía efectivos.

Se habla de estudiantes, de profesionales sanitarios, de hospitales, pero el gran ausente es la población a la que va destinada todo ese esfuerzo. Es en definitiva la población general la más ligada al sector sanitario ya que sin ella la sanidad no tiene sentido. Los mecanismos de acceso de la población al sistema sanitario, la desigualdad social frente al sistema sanitario, los efectos de la peculiar distribución de profesionales sanitarios sobre los diferentes estratos y clases sociales, y los tipos de asistencia y las relaciones médicopaciente, son elementos imprescindibles en un análisis del sector sanitario. Es importante además el estudio de la población porque incluso en el contexto estrecho del capitalismo sanitario la misma (y las mujeres más aún) cumple un papel principal como proveedora de asistencia sanitaria.

Una de las notas alarmantes es el imparable crecimiento del número de médicos. La equivocada idea de la escasez de médicos y la creencia en la profesión médica como carrera que lleva aparejada un elevado status socio-económico está muy arraigada en nuestro subconsciente colectivo. Ese gran número de médicos, que hoy ya están creando ciertas tensiones dentro de la profesión, planteó la necesidad de un numerus clausus en las Facultades de Medicina. Los criterios en los que se basa la selección no son los mejores y, a su vez, hay que tener presente que dicho numerus clausus no se puede convertir en una apología de la profesión médica, defendiendo su poder y prestigio al defender la idea de una estabilidad en su número. ¿Por qué se tienen que introducir elementos que reduzcan la competencia en el sector cuando en el conjunto de la sociedad y de las profesiones dicha competencia está presente $\mathrm{y}$, más aún, cuando la misma podría ser una de las formas de reducir el poder de la clase médica y ampliar su eficacia? Este elemento de competencia podría jugar el importante papel de racionalización del sistema sanitario. El mismo problema se plantea con el sector privado, que es de mala calidad y excesivamente caro. Si existe es porque el sector sanitario público lo permite. Permitir en este caso significa que el sector público no absorbe toda la demanda de servicios o que en muchos casos su asistencia es tan mala que obliga a la población a recurrir a servicios que «creen" son mejores. El problema no es acabar con el sector privado sino convertir al sector público en un sector competente y positivo. La existencia del sector sanitario privado, en una situación de completa competencia en el campo sanitario, podría, incluso, hacer de revulsivo para una racionalización y mejora del sector sanitario público.

No se puede dar por explicada la desigualdad sanitaria del país si únicamente se utiliza la vía de la distribución geográfica del personal sanitario. Es de gran utilidad para descubrir las desigualdades regionales, pero nos deja sólo en el preámbulo de las desigualdades sociales en el sector sanitario. Un análisis de loł mecanismos de acceso de la población a la asistencia, y de 
cómo tales mecanismos reflejan las diferencias sociales, nos permitiría comprender con más exactitud las desigualdades sanitarias del país.

La distribución de la población y del desarrollo pueden explicar la distribución de los profesionales sanitarios según los ejes norte-sur y este-oeste, pero no aclaran la abundancia de médicos especialistas en el sur ni la gran escasez en el norte, sobre todo en Galicia. La introducción de la variable propiedad y distribución de la tierra haría posible que la explicación fuera más completa. La parcelación de la tierra en el norte, y por tanto la abundancia de pequeños propietarios, hacen de la zona un área ideal para el médico general tradicional (médico-de-familia), pero no la hacen atractiva para el médico especialista. La existencia del latifundio en el sur, y la existencia de las grandes fortunas familiares atraen al especialista (que se convierte así en el médico-para-ricos), pero debido a la gran pobreza de la población no es atractivo para el médico-general.

Se parte del supuesto de que la escasez de médicos (en términos generales) en el sur se debe a la no existencia de Facultades de Medicina, y que para paliar este déficit sería necesaria la creación de Facultades. Cierto es que las zonas con Facultades tienen más médicos, y que las que tienen menos médicos no tienen Facultades, pero no menos cierto es que normalmente las Facultades se ubican en las áreas de gran desarrollo socio-económico. Por tanto, no es únicamente el factor de la existencia de Facultades el que atrae médicos, sino el conjunto de los factores que rodean el desarrollo socio-económico. La creación de nuevas Facultades, en zonas en las cuales el desarrollo económico no ha estimulado la existencia de infraestructuras sanitarias adecuadas, provocaría paro médico y migraciones de los médicos hacia áreas en las que hubiera demanda técnica de médicos. No es la creación de Facultades en las áreas pobres, sino el conseguir que dejen de ser pobres el que hará que, en el proceso de la reducción de las desigualdades regionales, las desigualdades sanitarias de carácter regional se vean amortiguadas.

\section{El poder de la desigualdad}

La distribución de médicos en España ha sido estudiada en detalle por Benjamín González, en un librito publicado por el Centro de Investigaciones Sociológicas en $1979^{12}$. La obra está enmarcada en la labor de investigación del autor durante los últimos años, realizada tanto en España como en los Estados Unidos. La importancia del tema en España es innegable, «ya que la asistencia sanitaria (preventiva y curativa) depende, en gran medida, del acceso equitativo de la población al capital médico humano. Querámoslo o no,

:2 Benjamí González Rodríguez, El capital humano en el sector sanitario: La distribución de médicos en España, Centro de Investigaciones Sociológicas, Madrid, 1979, 115 págs. 
los médicos, en la actualidad, son agentes de estratificación social» ${ }^{13}$. De su carácter netamente práctico surge la idea de su posible utilización en la planificación sanitaria, que tendría como fin la reducción de las desigualdades sanitarias ${ }^{14}$.

Se plantea un modelo matemático causal que permita: analizar las relaciones entre las variables independientes y las dependientes; establecer el orden causal de relación entre ellas; diferenciar entre los efectos directos $\mathrm{y}$ los indirectos, y estimar las influencias de grupos de variables independientes sobre la dependiente.

El recorrido metodológico se inicia haciendo la elección de las variables que se creen relevantes para explicar la distribución regional de médicos: renta per cápita, nivel de urbanización, número de camas en hospitales; nivel educativo, tasa de estudiantes de medicina como variables independientes, y, por supuesto, la tasa de médicos como variable dependiente. Las variables independientes se pueden agrupar a su vez en dos grupos de variables: las de carácter estructural y las de carácter sanitario. Se establecen así relaciones bivariables, realizando una ordenación por provincias y por regiones, para pasar a continuación a realizar un análisis multivariable. El modelo permite analizar el peso relativo de cada variable independiente sobre la variable dependiente, que varía según la región y la importancia del resto de las variables. Para ello se elabora una nueva reorganización de las regiones (basada en el resultado de los análisis bivariables): norte, sur, norte excluidos Madrid y Barcelona, total de España excluidos Madrid y Barcelona, y total de España.

La utilización de técnicas de regresión múltiple permiten manejar variables independientes simultáneamente. Aplicando la técnica de los mínimos cuadrados se obtienen cinco ecuaciones de regresión distintas, una por cada región. Las ecuaciones de regresión nos muestran el peso específico que cada variable independiente tiene sobre la variable dependiente, en esa zona concreta. La misma técnica podría utilizarse para la obtención de ecuaciones de regresión para cada provincia. Las ecuaciones de regresión representan matemáticamente el impacto de dichos procesos estructurales sobre la distribución de médicos.

Para demostrar que con las cinco variables propuestas se puede explicar gran parte de la variación existente en la distribución de los médicos se añade sucesivamente una variable más a la ecuación de regresión. Con ello se consigue a su vez apreciar los efectos de cada nueva variable sobre el conjunto de la ecuación.

A continuación, y dado que se parte de la hipótesis de que las razones de distribución son diferentes parà cada tipo de médico, se diseña una dis-

13 Ibidem, pág. 11.

" Un contrapunto de esta teoría sería el libro de Francisco Polo Finyo, El gran esclavo, el médico, Javier Morata, Madrid, 1929, 259 págs. 
tribución de los médicos en médico-general y especialista; y estos últimos se dividen en psiquiatras, cirujanos, internistas, ginecólogos y pediatras.

Todo este aparato estadístico y metodológico sirve de preámbulo al análisis de path (o de caminos), con el cual se llegan a conocer las influencias causales definitivas de las variables independientes sobre la dependiente. El objetivo de la técnica de path es resolver matemáticamente el cómo y en qué medida, dado un modelo estructural, toda variación en una variable puede ser explicada por la variación de determinadas variables independientes. En definitiva, la cantidad de influencia, poder causal o valor explicativo de las variables. El sistema permite descubrir los efectos directos e indirectos de cada variable independiente sobre la dependiente. A su vez hay ciertas variables que funcionan como dependientes y como independientes. Todo ello conduce a un modelo más bien complicado que permite diferenciar la influencia de cada variable sobre la tasa de médicos, para cada región española y para cada tipo de médicos.

La hipótesis de partida es netamente sociológica: los factores motivacionales individuales y sociales explican la distribución de los médicos en la geografía española, y estos últimos, los estructurales sociales básicos, son los que tienen el peso decisivo sobre la distribución. Aunque las motivaciones de tipo egoísta puedan tener importancia, son las motivaciones de índole económica, cultural. y profesional las que determinan la distribución. Por ello se utilizan las variables: renta, urbanización, educación, estudiantes de medicina, camas de hospitales; que son variables estructurales sociales básicas.

Existe una mala distribución de médicos en el territorio español, que afecta muy especialmente a las provincias con bajo nivel de desarrollo y básicamente rurales, en contraposición a las más ricas y urbanizadas. Esta deficiente regionalización de médicos se debe a la mala distribución de las variables socio-económicas, de tal manera que a un alto grado de presencia de variables socio-económicas irá asociado una alta presencia de médicos, y al revés.

Hay una relativa abundancia de médicos en el norte, sobre todo de médicos-generales, mientras que los especialistas se concentras básicamente en los vértices del hexágono del desarrollo. El sur presenta una alarmante escasez de médicos, con la única excepción de su alta concentración de especialistas. Frente a una concentración de las camas hospitalarias en el este, existe una clara escasez en el oeste. Todo ello nos presenta un mezzogiorno con gran escasez de médicos para la población general, y un norte, especialmente el noreste, relativamente bien dotado. Barcelona y Madrid son siempre grandes focos de atracción.

La renta es una variable importante para entender la preferencia geográfica de los médicos. En principio se espera que el impacto de la renta sea mayor en regiones pobres, debido a que son en ellas donde los factores socioeconómicos son más importantes. En cambio se supone que en las zonas des- 
arrolladas serán las variables de carácter sanitario las que tengan mayor importancia. Pero a pesar de ello los médicos se concentran en las zonas más ricas. Por tanto la relación entre renta y médicos será alta sobre todo en Galicia y en el sur. De ello podríamos deducir que las regiones con bajo nivel de renta tendrán pocos médicos, y viceversa.

Tal como ya planteó Jesús M. de Miguel en $1976^{15}$, la distribución de los médicos es netamente urbana; concentrándose el 78 por 100 de los médicos y farmacéuticos en zonas urbanas, y el 11 por 100 en zonas rurales. Cabe destacar, también, la gran concentración de médicos en la capital de provincia o en las grandes ciudades (como media hay 3,4 veces más médicos en capitales que en el resto de la provincia). Se puede observar un cierto grado de asociación entre la tasa de camas de hospitales y el número de médicos, aunque esta variable pueda estar influida por el contexto económico y urbano (esta relación no es excesivamente fuerte en España debido a la irracionalidad del sistema hospitalario). Consideramos al nivel de educación como un buen predictor del potencial médico. Pero la relación es mucho más estrecha cuando la educación es concretamente médica, pues existe una importante tendencia a ejercer donde se ha estudiado.

Empíricamente, con las cinco variables mencionadas, se puede explicar una gran parte de las variaciones existentes en la distribución de los médicos. Así pues, vemos que en el norte la variable más importante es el nivel de educación, seguida de estudiantes de medicina, y con la variable renta con carácter negativo (debido a su actual grado de desarrollo). Las variables urbanización y camas hospitalarias tienen poca importancia. En el sur la variable más importante es la de estudiante de medicina. El bajo nivel educativo general y la falta de urbanización no favorecen la práctica de la medicina.

Los elementos sociales y sanitarios responsables de la desigualdad social de recursos sanitarios siguen pautas diferentes según la zona. Se puede considerar que la variable que mejor explica la tasa de médicos es estudiantes de medicina, seguida del nivel general de educación. Renta y urbanización tienen poca influencia sobre el total de médicos, pero alta sobre los especialistas (ya que éstos tienden a ejercer en zonas de alto nivel económico). Sin embargo, la pauta que mejor explicaría la distribución de los médicos es la pauta de la distribución y propiedad de la tierra. Pequeños propietarios por un lado, y una estructura latifundista, explicarían el porqué de un mayor número de médicos generales en el norte, la gran concentración de especialistas en el sur (con una medicina casi exclusivamente para ricos), y la escasez de médicos-generales.

Se parte pues de la creencia de que es únicamente el médico el que forma el sector sanitario, y que explicando dicha profesión, su distribución, podemos explicar la distribución de la calidad y cantidad sanitaria del país. Sin

15 Jesús M. DE MIGUeL, La reforma sanitaria en España, op. cit., pág. 41. 
embargo, los otros profesionales sanitarios son también de vital importancia en el estudio del sector sanitario. La no inclusión en el análisis del resto de los profesionales sanitarios y de la estructuración de la población general ante el fenómeno sanitario hacen que se oscurezca la explicación de las desigualdades sanitarias.

A pesar del esfuerzo metodológico realizado hasta ahora en España para el análisis de la distribución de médicos (y médicas) los resultados son magros. No únicamente las variables que se introducen son las que explican el fenómeno de la distribución. La propiedad de la tierra, a la que se da la máxima importancia al finalizar el libro, no ha sido introducida en el proceso causal.

En un momento del libro el autor se plantea el elemento clase social como agente de la desigualdad social. Afirma que la profesión médica contribuye a esa desigualdad haciendo en muchos casos medicina-para-ricos y desatendiendo considerablemente el cuidado de los pobres. En un estudio de carácter netamente empírico es peligroso introducir argumentos de la importancia del presente sin la utilización de un aparato empírico que los respalde. El problema es uno de los cruciales en el análisis sociológico de las desigualdades, pero nunca ha sido objeto de un riguroso estudio. Estudio que junto al de la problemática de la distribución de los profesionales sanitarios nos explicaría claramente cuál es el tipo de sistema sanitario que existe. Queda pues todavía mucho trabajo sociológico para desempeñar el tema del poder médico, a pesar de estos buenos comienzos ${ }^{16}$.

16 Parte de este trabajo ha sido asumido en mi libro (en colaboración con otros médicos y sociólogos), Sociología de los ambulatorios: Análisis de la asistencia sanitaria primaria de la Seguridad Social, Ariel, Barcelona, 1979, 196 págs. 